\title{
Epidémiologie de la trypanosomose animale africaine chez les bovins dans le département du Korhogo (Côte d'Ivoire)
}

\author{
Ohoukou Marcel Boka ${ }^{1 *}$ Essehin Enock Jocelin Boka ${ }^{1,3}$ \\ Grégoire Yapi Yapi ${ }^{1}$ Seïdinan Ibrahima Traoré ${ }^{4}$ \\ Koffi Eric Kouamé ${ }^{2}$
}

\section{Mots-clés}

Bovin, Glossina, trypanosomose, épidémiologie, Côte d'Ivoire

Submitted: 10 May 2018

Accepted: 19 April 2019

Published: 12 July 2019

DOI: $10.19182 /$ remvt.31748

\section{Résumé}

Une étude transversale a été menée dans le département du Korhogo, au nord de la Côte d'Ivoire, dans la zone agropastorale de Katégué, afin de mieux connaître l'épidémiologie de la trypanosomose animale africaine (TAA) chez les bovins. L'étude a associé une enquête entomologique et une enquête parasitologique et s'est déroulée en saison des pluies, de juillet à octobre 2015. Les prospections entomologiques ont été réalisées à l'aide de pièges Vavoua posés dans 30 sites aux biotopes divers. Pour l'enquête parasitologique, 407 bovins ont été prélevés sur la base d'un échantillonnage aléatoire stratifié sans distinction de race, de sexe et d'âge. Les résultats ont montré la présence d'un vecteur majeur de la TAA, la mouche tsé-tsé Glossina palpalis gambiensis, avec une densité apparente globale de $0,9 \pm 3,0$ glossines par piège par jour. Une seule espèce de trypanosome a été identifiée, Trypanosoma vivax, avec des prévalences relativement faibles aussi bien chez les glossines (11 $\pm 5 \%$ ) que chez les bovins $(6 \pm 2 \%)$. Compte tenu de la gravité de la TAA chez les bovins, il s'avère nécessaire de sensibiliser les éleveurs du Korhogo, qui représente la principale zone d'élevage de bovins en Côte d'Ivoire, à la lutte contre les vecteurs de la TAA et à I'utilisation rationnelle des trypanocides.

- Comment citer cet article : Boka O.M., Boka E.E.J., Yapi G.Y., Traoré S.I., Kouamé K.E., 2019. Epidemiology of African animal trypanosomosis in cattle in Korhogo Department (Ivory Coast). Rev. Elev. Med. Vet. Pays Trop., 72 (2): 83-89, doi: 10.19182/remvt.31748
1. Université Alassane Ouattara (UAO), Centre d'entomologie médicale et vétérinaire (CEMV), Bouaké, Côte d'Ivoire.

2. Université Peleforo Gon Coulibaly (UPGC), UFR des sciences sociales, Département de géographie, Korhogo, Côte d'Ivoire.

3. Projet d'appui au développement de l'élevage en Côte d'Ivoire (PADE-CI), Antenne nord, Korhogo, Côte d'Ivoire.

4. Laboratoire national d'appui au développement agricole (Lanada), Laboratoire régional de Korhogo (LRK), Côte d'Ivoire.

* Auteur pour la correspondance

Tél. : +22507413075 / +224627915820

Email : marcelboka2@yahoo.fr

\section{INTRODUCTION}

La trypanosomose animale africaine (TAA) est une affection parasitaire d'importance vétérinaire causée par des hémoparasites flagellés du genre Trypanosoma. Elle affecte le bétail et peut évoluer sous forme chronique ou aiguë (OIE, 2012). Sous sa forme chronique, la TAA est responsable d'une forte dégradation de l'état général des animaux infectés qui, en maigrissant, deviennent des non-valeurs économiques, peu aptes aux travaux agricoles et sans aucune valeur monétaire à la réforme (Kamuanga et Kaboré, 2005). Sous sa forme aiguë, cette parasitose est mortelle en trois à quatre semaines (Vitouley, 2005).

En Côte d'Ivoire, tout le territoire est infesté par les mouches tsé-tsé (Hursey, 1985), vecteurs cycliques de cette hémoparasitose animale. De ce fait, plus de 1,2 million de bovins sont soumis au risque trypanosomien (Pokou et al., 2010) ; la TAA constitue une contrainte à la bonne production du bétail dans le pays. La maladie sévit surtout dans le nord (Douati et al., 1986 ; Acapovi-Yao et al., 2013 ; Djakaridja et al., 2014) 
où l'élevage bovin est très présent, mais les données épidémiologiques sur cette maladie dans la zone de Katégué sont inexistantes. L'objectif général de l'étude a ainsi été de disposer d'informations sur l'épidémiologie de la TAA chez les bovins de cette zone et trois objectifs spécifiques ont été retenus : a) inventorier les espèces de glossines présentes dans la zone et estimer leur abondance, b) évaluer le risque trypanosomien chez les glossines capturées et chez les bovins prélevés, et c) déterminer l'effet de la TAA sur l'état de santé des animaux prélevés.

\section{MATERIEL ET METHODES}

\section{Zone d'étude}

L'étude a été réalisée dans la zone agropastorale de Katégué $\left(09^{\circ} 33^{\prime} \mathrm{N}\right.$ et $05^{\circ} 51^{\prime}$ O) située dans le département du Korhogo, dans le nord de la Côte d'Ivoire, à environ 30 kilomètres de la ville de Korhogo (figure 1). Cette zone regroupe une dizaine de villages situés dans un rayon de 10 kilomètres environ autour du village de Katégué. La population appartient principalement au groupe linguistique Senoufo, bien que des Malinkés et des Peuls s'y trouvent également. Elle est majoritairement constituée d'agropasteurs ayant pour principales activités la culture du coton et l'élevage de bovins. Cette zone a été choisie parce qu'elle est caractérisée par la présence de nombreux troupeaux sédentaires de bovins et de bœufs de culture attelée. Ces animaux partagent les mêmes pâturages naturels et s'abreuvent, surtout en saison sèche, dans les portions non asséchées du principal cours d'eau, le Yoréloro, et dans le seul barrage agropastoral encore fonctionnel situé à Katégué.

Le paysage de cette zone est marqué par plusieurs types de formations végétales qui peuvent être des gîtes idéaux pour les glossines responsables de la TAA. Ce sont surtout des savanes boisées et herbacées parsemées de bois qui servent parfois de bois sacrés à la lisière des villages, ainsi que des galeries forestières situées le long du Yoréloro et des rivières affluentes, et le long du barrage agropastoral de Katégué.

Comme dans le reste du département, le climat est de type tropical soudanais avec une saison des pluies de mai à octobre et une saison sèche de novembre à avril, marquée par l'harmattan. La moyenne pluviométrique annuelle se situe entre 1100 et $1600 \mathrm{~mm}$ (Boko et al., 2016).

\section{Préenquête et échantillonnage}

Cette phase de l'étude a consisté en un recensement de tous les troupeaux sédentaires de bovins présents dans la zone d'étude. Les rencontres ont eu lieu dans tous les villages pendant une période d'environ 15 jours selon la disponibilité des éleveurs et des bouviers. Partant de ce recensement, un échantillonnage aléatoire stratifié fondé sur la taille des troupeaux de bovins a été réalisé.

Trois strates ont été identifiées : a) les troupeaux de petite taille (moins de 50 bovins) qui regroupaient $41 \%$ des troupeaux et $21 \%$ des animaux ; b) les troupeaux de taille moyenne (entre 50 et 100) qui regroupaient $38 \%$ des troupeaux et $36 \%$ des animaux; et c) les troupeaux de grande taille (plus de 100) qui regroupaient $21 \%$ des troupeaux et $43 \%$ des animaux. Les effectifs de bovins à prélever par strates ont été déterminés en tenant compte du poids de chaque strate dans la population échantillonnée. Quatre, sept et 14 bovins ont été prélevés par troupeau, respectivement dans les troupeaux de petite, moyenne et grande taille. Au total, ce sont 407 bovins de tous âges, des deux sexes et de différentes races qui ont été échantillonnés
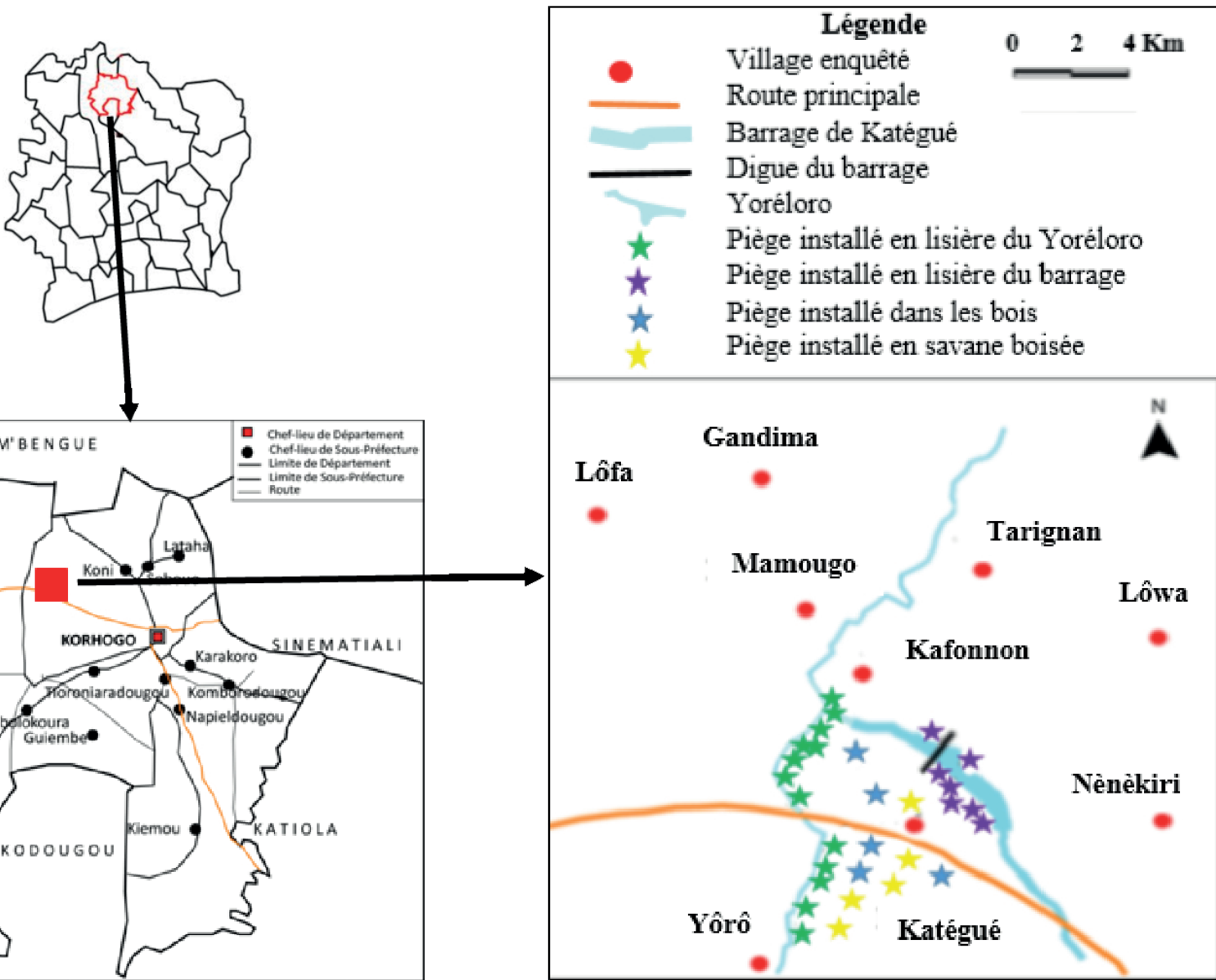

Source : Fond de carte, Kouassi14@un.orgt

Source : Image modifiée (Google Earth, 2015) Conception : Kouamé et Boka

Figure 1 : zone d'étude et localisation des sites de capture des glossines dans le nord de la Côte d'Ivoire. 
de façon aléatoire au sein des 56 troupeaux recensés dans la zone d'étude, soit environ $13 \%$ du cheptel total recensé (tableau I). Les sites de piégeage des glossines ont été aussi identifiés lors de la préenquête. C'étaient principalement des biotopes situés autour des lieux d'abreuvement et de pâturage du bétail.

\section{Enquête entomologique}

Les captures de glossines ont été réalisées en saison des pluies, du 4 au 9 août 2015. Trente sites diversifiés (galeries forestières, bois, savanes boisées) ont été prospectés durant cinq jours consécutifs à l'aide de pièges Vavoua (Laveissière et Grébaut, 1990). Chaque piège posé a été géoréférencé et les glossines y ont été récoltées toutes les 24 heures. Elles ont été transportées à l'antenne locale du Laboratoire national d'appui au développement agricole (Lanada) de Côte d'Ivoire, dans des tubes à essai préalablement étiquetés et bouchés avec du coton, et emballées dans une serviette mouillée afin de créer un microclimat favorable à leur survie. Les mouches ont été identifiées à l'aide de la clé établie par Pollock (1982) et seules les glossines en bon état (88 $\pm 5 \%$ des glossines capturées) ont été disséquées sous une loupe binoculaire (Optika) selon la technique décrite par Yoni et al. (2005).

Le proboscis (le couple labre-hypopharynx), les glandes salivaires et l'intestin moyen ont été séparés et préparés entre lame et lamelle pour la détection des trypanosomes au microscope optique. L'identification des espèces de trypanosomes a été faite selon leur localisation dans ces organes en utilisant la méthode de Lloyd et Johnson (1924).

\section{Enquête parasitologique}

Les données collectives et individuelles des animaux (type d'élevage, effectif, âge, type génétique, état corporel, date du dernier traitement antitrypanosomien) ont été enregistrées sur des fiches de collecte. La race des animaux a été identifiée sur la base des caractères morphologiques utilisés par Sokouri et al. (2007). Deux échantillons sanguins ont été prélevés sur chaque bovin, au niveau d'une veinule auriculaire, à l'aide de tubes capillaires à microhématocrite selon la technique décrite par Camus (1983). Une goutte de sang provenant de l'un de ces tubes capillaires était déposée sur une lame porte-objet préalablement identifiée pour la réalisation de frottis sanguin sur le terrain (OIE, 2012).

$\mathrm{Au}$ laboratoire, les tubes capillaires ont été centrifugés pendant cinq minutes à 12000 tours par minute. La lecture de l'hématocrite a été effectuée avec le disque de lecture de la centrifugeuse (Hettich Haematokrit 210). Un hématocrite d'une valeur inférieure ou égale à $25 \%$ était considéré comme révélateur d'une anémie (Dao et al., 2008 ; Ingabiré, 2009).

Les frottis réalisés sur le terrain ont été fixés avec du méthanol puis colorés dans un bac contenant une solution de Giemsa à $10 \%$. Ils ont été ensuite observés au microscope optique à l'objectif 100 en vue d'identifier les trypanosomes suivant les critères morphologiques (taille et position du kinétoplaste, forme de l'extrémité postérieure, présence ou non d'un flagelle libre, présence ou non d'une membrane ondulante, taille du trypanosome) proposés par Hoare (1972) et l'OIE (2012).

\section{Analyse des données}

L'abondance des glossines est traduite par le calcul de la densité apparente par piège et par jour (DAP) selon la formule suivante :

$\mathrm{DAP}=\frac{\mathrm{nb} \cdot \text { de glossines capturées }}{(\mathrm{nb} \cdot \text { de pièges }) \times(\mathrm{nb} \cdot \text { de jours de capture })}$

Les prévalences trypanosomiennes ont été calculées chez les glossines disséquées et chez les bovins prélevés selon les formules suivantes :

Prévalence $($ glossines $)=\frac{\text { nb. de glossines infestées }}{\text { nb. de glossines disséquées }} \times 100$

Prévalence (bovins) $=\frac{\text { nb. de bovins parasités }(\text { positifs })}{\text { nb. total de bovins prélevés }} \times 100$

\section{Tableau I}

Echantillonnage des bovins en fonction des villages et de la taille des troupeaux dans le nord de la Côte d'Ivoire

\begin{tabular}{|c|c|c|c|c|c|c|c|c|}
\hline \multirow[t]{3}{*}{ Village } & \multirow{3}{*}{$\begin{array}{l}\text { Nb. de } \\
\text { troupeaux } \\
\text { / village }\end{array}$} & \multicolumn{6}{|c|}{ Nombre de bovins par troupeau } & \multirow{3}{*}{$\begin{array}{l}\text { Nb. total } \\
\text { de bovins } \\
\text { prélevés }\end{array}$} \\
\hline & & \multicolumn{2}{|c|}{$\begin{array}{c}<50(\text { strate } 1) \\
n_{1}=647\end{array}$} & \multicolumn{2}{|c|}{$\begin{array}{c}50-100 \text { (strate } 2) \\
n_{2}=1146\end{array}$} & \multicolumn{2}{|c|}{$\begin{array}{c}>100 \text { (strate } 3) \\
n_{3}=1339\end{array}$} & \\
\hline & & $\begin{array}{l}\mathrm{Nb} \text {. de } \\
\text { troupeaux }\end{array}$ & $\begin{array}{l}\text { Nb. de } \\
\text { bovins } \\
\text { prélevés }\end{array}$ & $\begin{array}{l}\mathrm{Nb} \text {. de } \\
\text { troupeaux }\end{array}$ & $\begin{array}{l}\text { Nb. de } \\
\text { bovins } \\
\text { prélevés }\end{array}$ & $\begin{array}{l}\mathrm{Nb} \text {. de } \\
\text { troupeaux }\end{array}$ & $\begin{array}{l}\text { Nb. de } \\
\text { bovins } \\
\text { prélevés }\end{array}$ & \\
\hline Katégué & 10 & 4 & 16 & 4 & 28 & 2 & 28 & 72 \\
\hline Kafonnon & 2 & 2 & 8 & 0 & 0 & 0 & 0 & 8 \\
\hline Nènèkiri & 11 & 3 & 12 & 3 & 21 & 5 & 70 & 103 \\
\hline Lôwa & 7 & 5 & 20 & 1 & 7 & 1 & 14 & 41 \\
\hline Tarignan & 1 & 1 & 4 & 0 & 0 & 0 & 0 & 4 \\
\hline Mamougou & 8 & 2 & 8 & 4 & 28 & 2 & 28 & 64 \\
\hline Gandiman & 6 & 2 & 8 & 4 & 28 & 0 & 0 & 36 \\
\hline Lôfa & 7 & 3 & 12 & 3 & 21 & 1 & 14 & 47 \\
\hline Yôrô & 4 & 1 & 4 & 2 & 14 & 1 & 14 & 32 \\
\hline Total & 56 & $\begin{array}{c}23 \\
\left(\mathrm{~m}_{1}\right)\end{array}$ & 92 & $\begin{array}{c}21 \\
\left(\mathrm{~m}_{2}\right)\end{array}$ & 147 & $\begin{array}{c}12 \\
\left(\mathrm{~m}_{3}\right)\end{array}$ & 168 & 407 \\
\hline
\end{tabular}

Calcul du nombre de bovins à prélever par troupeau : $\mathrm{M}_{\mathrm{i}}=\left(\mathrm{n}_{\mathrm{i}} / \mathrm{m}_{\mathrm{i}}\right) \times \mathrm{T} / \mathrm{N}$; où $\mathrm{n}_{\mathrm{i}}$ représente le nombre de bovins recensés par strate, $\mathrm{m}_{\mathrm{i}}$ le nombre de troupeaux recensés par strate, et $\mathrm{T}$ l'effectif théorique de bovins à échantillonner parmi un total de $\mathrm{N}$ bovins selon la table d'échantillonnage (350 arrondi à 400), avec une marge d'erreur de $5 \%$, $\mathrm{p}=0,5 ;$ un niveau de confiance $=95 \% ; \mathrm{N}=3132 ; \mathrm{T}=400 \rightarrow \mathrm{T} / \mathrm{N}=0,13$. Ainsi, $\mathrm{M}_{1}=4 ; \mathrm{M}_{2}=7 ; \mathrm{M}_{3}=14$ 
Le test du Chi carré $\left(\chi^{2}\right)$ de Pearson a été utilisé pour la comparaison des prévalences. Les moyennes des DAP ont été comparées à l'aide d'une analyse de variance (test Anova). Le seuil de significativité de toutes les analyses a été fixé à $5 \%$.

\section{RESULTATS}

\section{Résultats entomologiques}

\section{Abondance et identification des glossines}

L'enquête entomologique a permis de récolter 138 glossines, soit une DAP globale de 0,9 $\pm 3,0$ glossines par piège par jour. Les moyennes des DAP étaient significativement variables selon les biotopes prospectés (tableau II). Les glossines étaient toutes de la sous-espèce Glossina palpalis gambiensis. Le tri de ces glossines par sexe a permis de dénombrer 81 femelles et 57 mâles soit un sex-ratio (femelle sur mâle) de 1,42.

\section{Infestation des glossines}

Au total 122 glossines ont été disséquées et seules 14 d'entre elles étaient infestées - prévalence globale de $11 \pm 5 \%$-, toutes au niveau du proboscis (les intestins et les glandes salivaires n'étaient jamais infestés) ce qui correspondait à Trypanosoma vivax. Les glossines femelles étaient plus infectées que les mâles mais de façon non significative (tableau III).

\section{Résultats parasitologiques}

\section{Caractéristiques des bovins prélevés}

Trois types de bovins ont été identifiés dans la zone d'étude : des taurins $(15,23 \%)$, représentés par les races Baoulé $(9,10 \%)$ et N'dama $(6,14 \%)$ qui sont censées être trypanotolérantes ; des zébus (21,13\%), principalement des races sahéliennes ; et des métis $(63,64 \%)$ issus de croisements divers (tableau IV). Le cheptel prélevé était dominé par des femelles âgées de plus de trois ans.

\section{Infection des bovins}

Après analyse des 407 frottis colorés, 26 ont été considérés comme positifs, soit une prévalence globale de $6 \pm 2 \%$. Trypanosoma vivax a été le seul trypanosome identifié. Ce trypanosome a été détecté dans toutes les tranches d'âge avec toutefois de faibles prévalences chez les jeunes bovins (tableau V). Cette différence était significative $\left(\chi^{2}=8,4182 ; \mathrm{p}<0,02\right)$.

\section{Relation hématocrite et infection des bovins}

L'hématocrite moyen des animaux prélevés était de $31 \pm 6 \%$. Dans l'échantillon étudié, $81 \pm 3 \%$ des bovins avaient un hématocrite supérieur à $25 \%$, et $19 \pm 3 \%$ un hématocrite inférieur ou égal à $25 \%$, avec une moyenne de $22 \pm 2 \%$. Les bovins porteurs de trypanosomes avaient un hématocrite moyen $(28 \pm 7 \%)$ inférieur à celui des animaux trouvés négatifs ( $32 \pm 6 \%$ ), mais la différence n'était pas significative. De façon générale, une prévalence plus élevée de cas d’anémie (77 ; $19 \pm 3 \%)$ que d'infection trypanosomienne $(26 ; 6 \pm 2 \%)$ a été observée. Cette différence était très significative $\left(\chi^{2}=28,9 ; p<0,001\right)$.

\section{DISCUSSION}

La prospection entomologique a révélé la présence de la tsé-tsé dans la zone d'étude. Cependant, la faible récolte de glossines, traduite par une DAP globale de $0,9 \mathrm{~g} / \mathrm{p} / \mathrm{j}$, pourrait s'expliquer par l'utilisation

\section{Tableau II}

Densité apparente (DAP) des glossines capturées en fonction des biotopes prospectés dans le nord de la Côte d'Ivoire

\begin{tabular}{|c|c|c|c|c|c|c|}
\hline $\begin{array}{l}\text { Site de } \\
\text { piégeage }\end{array}$ & $\begin{array}{l}\mathrm{Nb} \text {. de } \\
\text { pièges } \\
\text { posés }\end{array}$ & $\begin{array}{l}\text { Nb. de } \\
\text { glossines } \\
\text { capturées }\end{array}$ & DAP & $F$ & ddl & $\mathbf{P}$ \\
\hline $\begin{array}{l}\text { Lisière du } \\
\text { Yoréloro }\end{array}$ & 13 & 104 & $1,6 \pm 3,8$ & \multirow{5}{*}{3,89} & \multirow{5}{*}{$\begin{array}{c}K 1=3 \\
K 2=26\end{array}$} & \multirow{5}{*}{$-0,025$} \\
\hline $\begin{array}{l}\text { Lisière du } \\
\text { barrage }\end{array}$ & 7 & 25 & $0,7 \pm 2,0$ & & & \\
\hline Bois & 5 & 7 & $0,3 \pm 1,5$ & & & \\
\hline $\begin{array}{l}\text { Savane } \\
\text { boisée }\end{array}$ & 5 & 2 & $0,1 \pm 0,8$ & & & \\
\hline $\begin{array}{l}\text { Abondance } \\
\text { globale }\end{array}$ & 30 & 138 & $0,9 \pm 3,0$ & & & \\
\hline
\end{tabular}

$<0,025$ : effet significatif

\section{Tableau III}

Infection des glossines disséquées en fonction du sexe (nord de la Côte d'Ivoire)

\begin{tabular}{lccccc} 
& $\begin{array}{c}\text { Nb. de G. } \\
\text { disséquées }\end{array}$ & $\begin{array}{c}\text { Nb. de G. } \\
\text { infectées }\end{array}$ & $\begin{array}{c}\text { Prév. } \\
(\%)\end{array}$ & $\chi^{2}$ & P \\
\hline Femelle & 66 & 11 & $16 \pm 9$ & & \\
Mâle & 56 & 3 & $5 \pm 5$ & 3,81 & $>0,05$ \\
$\begin{array}{l}\text { Prév. } \\
\text { globale }\end{array}$ & 122 & 14 & $11 \pm 5$ & & \\
\hline
\end{tabular}

G. : glossines ; Prév. : prévalence ; 0,05 : effet non significatif

\section{Tableau IV}

Répartition des bovins prélevés en fonction de la race, du sexe et de la tranche d'âge (nord de la Côte d'Ivoire)

\begin{tabular}{|c|c|c|c|c|c|c|c|}
\hline \multirow[t]{2}{*}{ Race } & \multicolumn{3}{|c|}{ Sexe } & \multicolumn{3}{|c|}{ Tranche d'âge } & \multirow[t]{2}{*}{ Total } \\
\hline & $\mathbf{F}$ & M & Total & $<1$ an & $1-3$ ans & $>3$ ans & \\
\hline N'dama & 22 & 3 & 25 & 3 & 4 & 18 & 25 \\
\hline Baoulé & 33 & 4 & 37 & 1 & 7 & 29 & 37 \\
\hline Zébus & 47 & 39 & 86 & 11 & 31 & 44 & 86 \\
\hline Métis & 197 & 62 & 259 & 28 & 105 & 126 & 259 \\
\hline Total & 299 & 108 & 407 & 43 & 147 & 217 & 407 \\
\hline Fréquence (\%) & $73 \pm 4$ & $27 \pm 4$ & & $11 \pm 2$ & $36 \pm 4$ & $53 \pm 4$ & \\
\hline
\end{tabular}




\section{Tableau V}

Prévalence de la trypanosomose animale africaine chez les bovins en fonction de la race, du sexe et de la tranche d'âge (nord de la Côte d'Ivoire)

\begin{tabular}{lccccc} 
& $\begin{array}{c}\text { Nb. de B. } \\
\text { prélevés }\end{array}$ & $\begin{array}{c}\text { Nb. de B. } \\
\text { parasités }\end{array}$ & Prév. (\%) & $\chi^{\mathbf{2}}$ & P \\
Race & & & & & \\
N'dama & 25 & 1 & $4 \pm 7$ & & \\
Baoulé & 37 & 0 & $0 \pm 0$ & 3,20 & $>0,05$ \\
Zébus & 86 & 6 & $7 \pm 5$ & & \\
Métis & 259 & 19 & $7 \pm 3$ & & \\
Sexe & & & & & \\
Femelle & 299 & 19 & $6 \pm 2$ & $<0,01$ & $>0,05$ \\
Mâle & 108 & 7 & $6 \pm 4$ & & \\
Age & & & & & \\
$<1$ an & 43 & 1 & $2^{\text {ab }} \pm 4$ & & \\
$1-3$ ans & 147 & 4 & $2^{\mathrm{a}} \pm 2$ & 8,42 & $<0,02$ \\
$>3$ ans & 217 & 21 & $9^{b} \pm 3$ & & \\
\hline
\end{tabular}

B. : bovins ; Prév. : prévalence ; >0,05 : effet non significatif ; $<0,02$ : effet significatif

${ }^{\mathrm{a}, \mathrm{b}}$ Les valeurs sur une même colonne suivies de la même lettre ne diffèrent pas significativement au seuil de $5 \%$.

d'un seul type de piège et l'étude de seulement 30 sites de piégeage. Des études antérieures ont montré que l'association de plusieurs types de pièges et l'ajout d'attractif pouvaient amplifier les captures des glossines (Bouyer et al., 2005 ; Zinga Koumba et al., 2013). De plus, toute la zone d'étude est une zone d'intense activité agricole dominée par la culture du coton. L'utilisation intensive, associée à cette culture, d'insecticides et d'herbicides (dont certains comme le carbamate ont une action insecticide) pourrait être l'une des causes de la réduction de la densité des glossines. Ces pesticides, pour la plupart des insecticides non sélectifs, détruiraient les insectes présents dans les champs pulvérisés et aux alentours. Néanmoins, la DAP globale obtenue lors de cette étude était similaire à celle obtenue par Acapovi-Yao et al. (2013) dans le département voisin du Sinématiali $(0,8 \mathrm{~g} / \mathrm{p} / \mathrm{j})$. La faible capture de glossines pourrait enfin aussi être due à la plus forte dispersion des glossines en saison des pluies, période à laquelle elles ne restent pas concentrées dans les forêtsgaleries comme mentionné par Djohan et al. (2015b).

Glossina palpalis gambiensis, la seule sous-espèce de glossine capturée, est d'ailleurs présente dans le Korhogo depuis plus de trois décennies (Laveissière et Challier, 1981 ; Douati et al., 1986 ; Acapovi-Yao et al., 2013). La capture de glossines du seul sous-genre Nemorhina pourrait être la conséquence de l'anthropisation du milieu. Selon Courtin et al. (2009), l'anthropisation provoque en savane la dégradation du couvert végétal et la quasi-disparition de la faune sauvage, indispensable à la présence des glossines de savane (sous-genre Glossina) et favorable aux fortes densités de glossines riveraines (sous-genre Nemorhina). En outre, la survie des glossines dans un biotope donné dépend de la présence conjuguée de plusieurs facteurs environnementaux propices (Laveissière et al., 2000 ; Solano et al., 2010). Dans la zone d'étude, ces conditions favorables semblent se localiser actuellement dans les galeries forestières présentes à la lisière des points d'eau (cours d'eau, barrage pastoral) qui sont les lieux où le plus grand nombre de glossines a été capturé. Ces biotopes sont d'ailleurs les lieux de prédilection des espèces du sous-genre Nemorhina dont G.p. gambiensis (de La Rocque, 2003). Les glossines femelles étaient plus nombreuses que les mâles, comme rapporté par d'autres auteurs (Laveissière et al., 2000 ; Bosson-Vanga et al., 2012) qui l'expliquent par la plus grande longévité des glossines femelles.
Trypanosoma vivax a été la seule espèce de trypanosome identifiée dans les glossines. Djohan et al. (2015a) avaient quant à eux identifié en 2012 trois espèces de trypanosomes ( $T$. congolense type savane, T. vivax, T. brucei s.l.) à Kafolo, dans la région du Tchologo située également dans le nord de la Côte d'Ivoire. Cette divergence pourrait s'expliquer par le fait que ces auteurs ont utilisé des techniques moléculaires pour identifier les trypanosomes dans les glossines alors que ces méthodes n'ont pas été utilisées pour la présente étude. Les glossines femelles étaient plus infestées que les mâles par les trypanosomes, bien que non significativement. D'autres études mettent en évidence une infection plus grande des glossines femelles, comme celle de Allou et al. (2009) concernant G. palpalis au sud de la Côte d'Ivoire. Maudlin et al. (1991) l'expliquent par le fait que la capacité vectorielle des glossines femelles est trois fois plus importante que celle des mâles à cause de leur plus grande longévité et de leur plus grande faculté à se nourrir à intervalles plus rapprochés que les mâles.

La grande présence de bovins métis dans le cheptel échantillonné pouvait s'expliquer par l'absence de schéma d'amélioration génétique de la part des éleveurs ou, à l'inverse, par des stratégies délibérées de croisement des différentes races afin de bénéficier des caractéristiques des deux espèces bovines présentes (taurine et zébu). Cette situation se traduisait par l'hétérogénéité des troupeaux où des croisements désordonnés entre les espèces et les races engendraient des métissages très importants, comme le montrent Sokouri et al. (2007) dans cette région du nord de la Côte d'Ivoire.

Généralement, trois espèces de trypanosomes pathogènes (Trypanosoma vivax, T. congolense, T. brucei s.l.) infectent le bétail (de La Rocque, 2003). Dans notre étude, T. vivax a été la seule espèce identifiée chez les bovins prélevés, comme elle a été la seule observée dans les glossines capturées. Selon Cuisance et al. (2003), ce trypanosome est le plus fréquemment rencontré chez les bovins. La non-identification des autres espèces de trypanosome chez les bovins pourrait cependant être due à la technique d'identification utilisée. En effet, bien que spécifiques, les techniques parasitologiques sont peu sensibles pour le diagnostic des infections actives du bétail par les trypanosomes, surtout lorsque les animaux sont en phase chronique d'infection (Gardiner, 1989 ; Desquesnes et Tresse, 1996). Cette hypothèse semble être confirmée par Koffi et al. (2014) qui ont mis en évidence, par la technique d'amplification en chaîne par polymérase (PCR), les trois espèces de trypanosomes pathogènes du bétail, avec une dominance de T. brucei s.l., dans le département de Bouna au nord-est de la Côte d'Ivoire. N'Djétchi et al. (2017) rapportent des observations similaires également avec la PCR dans le centre-ouest du pays.

La faible prévalence trypanosomienne observée chez les bovins prélevés pourrait être due à l'utilisation intensive d'insecticides ou d'acaricides, homologués ou non, chez les animaux pour la lutte contre les tiques. En effet, la présence de nombreuses tiques sur les bovins oblige les éleveurs à utiliser très fréquemment ces produits pour traiter les animaux parasités. Ces insecticides agissent comme des répulsifs qui limitent le contact entre les bovins traités et les insectes hématophages. L'utilisation en routine des trypanocides par les éleveurs pour la prévention ou la lutte contre la TAA contribuerait également à cette faible prévalence. Selon Cuisance et al. (2003), les trypanocides représentent $44 \%$ du marché total des médicaments vétérinaires en Afrique subsaharienne. D'ailleurs, les antiparasitaires, dont les trypanocides, constituent les médicaments vétérinaires les plus vendus en Côte d'Ivoire (Yapo, 2011). Une prévalence similaire à celle observée dans notre étude $(6 \pm 2 \%)$ a toutefois été obtenue par Djakaridja et al. (2014) chez les bovins dans le nord de la Côte d'Ivoire (6,9\%).

L'infection trypanosomienne a été observée dans toutes les tranches d'âge avec de plus faibles prévalences chez les jeunes bovins. Ce résultat pourrait s'expliquer par le fait que les animaux les plus jeunes bénéficiaient, dans les élevages visités, d'un meilleur suivi sanitaire et médical. 
De plus, la plupart des veaux de moins d'un an étaient maintenus au piquet toute la journée ou pâturaient aux alentours des parcs situés à la lisière des villages. Ils étaient par conséquent moins en contact avec les vecteurs de la TAA.

Le mode d'élevage adopté par les éleveurs de la zone et la grande présence de bovins adultes dans les troupeaux pourraient également contribuer à expliquer ce résultat. En effet, la raréfaction des ressources pastorales - surtout en saison d'hivernage comme rapporté par les éleveurs -, causée par l'accroissement des surfaces cultivées (entre autres vergers de manguiers, d'anacardiers, champs de coton), obligeait le bétail à pâturer dans des espaces restreints situés autour des points d'eau (cours d'eau, barrage pastoral) et dans les rares jachères non encore exploitées. Le grand attroupement du bétail dans ces zones pourrait être une source importante d'infection des animaux, favorisée par le contact direct avec les arthropodes vecteurs. Selon les travaux de Torr et al. (2007) sur l'effet de la taille et de la composition du troupeau sur les taux de piqûres individuels par les mouches tsé-tsé, les bovins les plus âgés présentent plus de « risque » d'être piqués que les plus jeunes. Cependant, nos résultats ne concordent pas avec ceux de Tanenbe et al. (2010), et Acapovi-Yao et al. (2016) qui rapportent que les jeunes bovins sont plus infectés par les trypanosomes que les adultes, mais de façon non significative.

La prévalence significativement plus élevée des anémies $(19 \pm 3 \%)$ que des infections trypanosomiennes $(6 \pm 2 \%)$ pourrait être expliquée par les limites de la technique d'étalement mince de sang utilisée pour l'identification des trypanosomes. Cette technique, bien que relativement sensible (OIE, 2012), n'aurait pas permis de détecter tous les cas positifs. Mais il est également possible que cette différence ait été due à la présence d'autres facteurs anémiants, notamment les hémoparasites transmis par les tiques, comme cela a été rapporté par Djakaridja et al. (2014) dans le nord de la Côte d'Ivoire.

\section{- CONCLUSION}

Cette étude visait à mieux connaître l'épidémiologie de la TAA chez les bovins dans le département du Korhogo. Glossina palpalis gambiensis, l'un des vecteurs de cette hémoparasitose animale, a été le seul vecteur identifié dans la zone d'étude avec une DAP globale de 0,9 $\pm 3,0$ $\mathrm{g} / \mathrm{p} / \mathrm{j}$. Trypanosoma vivax a été quant à lui le seul trypanosome identifié avec des prévalences relativement faibles à la fois chez les glossines $(11 \pm 5 \%)$ et chez les bovins $(6 \pm 2 \%)$. Une prévalence plus élevée des cas d'anémie $(19 \pm 3 \%)$ a également été observée. Malgré les faibles prévalences enregistrées, le risque de transmission de la TAA chez les bovins de la région demeure. Le fort taux de métissage des bovins dans les troupeaux et l'élevage en divagation à la recherche constante de pâturages augmenteraient ce risque. Il serait nécessaire de sensibiliser les éleveurs de ce département, principale zone d'élevage de bovins en Côte d'Ivoire, à la lutte contre les vecteurs de la TAA et à l'utilisation rationnelle de trypanocides.

\section{Déclaration des contributions des auteurs}

OMB et GYY ont conçu et planifié l'étude ; OMB a supervisé les opérations de collecte sur le terrain et l'analyse des échantillons au laboratoire ; EEJB et SIT ont participé à la collecte et à l'analyse des données au laboratoire ; OMB, EEJB et KEK ont effectué les analyses statistiques et réalisé la figure 1 ; EEJB a rédigé la première version du manuscrit ; OMB et GYY ont révisé le manuscrit.

\section{REFERENCES}

Acapovi-Yao G., Cissé B., Mavoungou J.F., Kohagné Tongue L., Coulibaly N., 2013. Situation de la trypanosomose bovine dans les principales régions d'élevage au nord de la Côte d'Ivoire après la crise socio-militaire. Rev. Afr. Santé Prod. Anim., 11 (1) : 17-22
Acapovi-Yao G., Cissé B., Zinga Koumba C.R., Mavoungou J.F., 2016. Infections trypanosomiennes chez les bovins dans des élevages de différents départements en Côte d'Ivoire. Rev. Méd. Vét., 167 (9-10) : 289-295

Allou K., Acapovi-Yao G., Kaba D., Bosson-Vanga H., Solano P., N'goran K.E., 2009. Chorologie et infection par les trypanosomes de Glossina palpalis dans la forêt du Banco et ses reliques, Abidjan (Côte d'Ivoire). Parasite, 16 : 289 295, doi : 10.1051/parasite/2009164289

Boko A.N.N, Cissé G., Koné B., Dedy S.F., 2016. Croyances locales et stratégies d'adaptation aux variations climatiques à Korhogo (Côte d'Ivoire). Tropicultura, 34 (1) : 40-46

Bosson-Vanga A.H., Acapovi-Yao G., Kaba D., Dofini F., Coulibaly B., N'dri L., Koné M., 2012. Infection de Glossina palpalis palpalis par les trypanosomes le long du fleuve Comoé dans la région d'Abengourou (Côte d'Ivoire). J. Sci. Pharm. Biol., 13 (1) : 31-37

Bouyer J., Desquesnes M., Kaboré I., Dia M.L., Gilles J., Yoni W., Cuisance D., 2005. Les trypanosomoses animales africaines. Le piégeage des insectes vecteurs. Cirdes, Bobo Dioulasso, Burkina Faso, 12 p. (Santé animale en Afrique de l'Ouest ; 20)

Camus E., 1983. Diagnostic de la trypanosomose bovine sur le terrain par la méthode de centrifugation hématocrite. Rev. Sci. Tech. Off. Int. Epizoot., 2 (3) : 751-769, doi : 10.20506/rst.2.3.130

Courtin F., Sidibé I., Rouamba J., Jamonneau V., Gouro A., Solano P., 2009. Impacts des évolutions démographiques et climatiques sur la répartition spatiale des hommes, des tsé-tsé et des trypanosomoses en Afrique de l'Ouest. Parasite, 16 (1) : 3-10, doi : 10.1051/parasite/2009161003

Cuisance D., Itard J., Desquesnes M., Frezil J.-L., de La Rocque S., 2003. Trypanosomoses. Epidémiologie. In : Principales maladies infectieuses et parasitaires du bétail. Europe et régions chaudes. Tome 2 : Maladies bactériennes, mycoses, maladies parasitaires (éds. Lefèvre P.C., Blancou J., Chermette R.). Lavoisier, Paris, France, 1627-1650

Dao B., Hendrickx G., Sidibé I., Belem A.M.G., de La Rocque S., 2008. Impact of drought and degradation of protected areas on the distribution of bovine trypanosomoses and their vectors in the Oti catchment basin of Northern Togo. Rev. Elev. Med. Vet. Pays Trop., 61 (3-4): 153-160, doi: 10.19182/ remvt.9982

De La Rocque S., 2003. Epidémiologie des trypanosomoses africaines. Analyse et prévision du risque dans des paysages en transformation. Courr. Environ. INRA, $49: 80-86$

Desquesnes M., Tresse L., 1996. PCR sensitivity for Trypanosoma vivax detection with several methods of blood sample preparations. Rev. Elev. Med. Vet. Pays Trop., 49 (4): 322-327, doi: 10.19182/remvt.9504

Djakaridja B., Yao Kouassi P., Gragnon Biego G., Acapovi-Yao G., Mavoungou J.F., N'goran Kouakou E., 2014. Situation épidémiologique des hémoparasites des bovins dans deux zones d'élevage de la Côte d'Ivoire : cas des anciennes régions des Savanes et de la vallée du Bandama. Rev. Méd. Vét., 165 (9-10) : 297-303

Djohan V., Kaba D., Rayaissé J.-B., Dayo G.-K., Coulibaly B., Salou E., Dofini F., et al., 2015a. Detection and identification of pathogenic trypanosome species in tsetse flies along the Comoé River in Côte d'Ivoire. Parasite, 22 (18): 1-7, doi : 10.1051/parasite/2015018

Djohan V., Kaba D., Rayaissé J.-B., Salou E., Coulibaly B., Dofini F., Kouadio K.A.M., et al., 2015b. Spatial and temporal diversity of tsetse flies along Comoe River in Côte d'Ivoire. Rev. Elev. Med. Vet. Pays Trop., 68 (1): 39-44, doi: 10.19182/remvt.20575

Douati A., Kupper W., Kotia K., Badou K., 1986. Tsetse control (Glossina: Diptera, Muscidae) by means of screens and traps (static methods): two years control evaluation (Sirasso, north of Côte d'Ivoire). Rev. Elev. Med. Vet. Pays Trop., 39 (2): 213-219, doi: 10.19182/remvt.8588

Gardiner P.R., 1989. Recent studies of the biology of Trypanosoma vivax. Adv. Parasitol., 28: 229-317, doi: 10.1016/S0065-308X(08)60334-6

Hoare C.A., 1972. The trypanosomes of mammals. A zoological monograph. Blackwell Scientific, Oxford, UK, 749 p.

Hursey B.S., 1985. Lutte contre les glossines en Afrique. Rev. Sci. Tech. Off. Int. Epizoot., 4 (2) : 299-310, doi : 10.20506/rst.4.2.201

Ingabiré C., 2009. Trypanosomose bovine au Ghana : prévalences sérologique et parasitologique; intérêt de l'utilisation du système d'information géographique. Mém. Master II Santé publique vétérinaire, EISMV, Dakar, Sénégal, $30 \mathrm{p}$.

Kamuanga M., Kaboré I., 2005. La lutte contre les glossines dans la zone agropastorale de Yalé (Burkina Faso) : résultats d'enquêtes socioéconomiques. Tropicultura, 23 (3) : 146-153 
Koffi M., Kouadio K.I., Sokouri D.P., Wognin M.T., N'Guetta A.S.P., 2014. Molecular characterization of trypanosomes isolated from naturally infected cattle in the "Pays Lobi" of Côte d'Ivoire. J. Appl. Biosci., 83: 7570-7578, doi: 10.4314/jab.v83i1.10

Laveissière C., Challier A., 1981. La répartition des glossines en Côte d'Ivoire. Cartes à 1/2 $000000^{\mathrm{e}}$. Orstom, Paris, France, 33 p. (Notice explicative ; 89)

Laveissière C., Grébaut P., 1990. Recherches sur les pièges à glossines (Diptera : Glossinidae). Mise au point d'un modèle économique : le piège "Vavoua ». Trop. Méd. Parasitol., 41 : 185-192

Laveissière C., Grébaut P., Herder S., Penchenier L., 2000. Les glossines vectrices de la trypanosomiase humaine africaine. IRD/OCEAC, Yaoundé, Cameroun, $246 \mathrm{p}$.

Lloyd L., Johnson W.B., 1924. The trypanosome infections of tsetse flies in Northern Nigeria and a new method of estimation. Bull. Entomol. Res. 14 (3): 265-288, doi: 10.1017/S0007485300028352

Maudlin I., Welburn S.C., Milligan P., 1991. Salivary gland infection: a sexlinked recessive character in tsetse? Acta Trop., 48 (1): 9-15, doi: 10.1016/0001-706X(90)90060-D

N’Djetchi M.K., Ilboudo H., Koffi M., Kaboré J., Kaboré J.W., Kaba D., Courtin F. et al., 2017. The study of trypanosome species circulating in domestic animals in two human African trypanosomiasis foci of Côte d'Ivoire identifies pigs and cattle as potential reservoirs of Trypanosoma brucei gambiense. PLoS Negl. Trop. Dis., 11 (10): e0005993, doi: 10.1371/journal.pntd.0005993

OIE, 2012. Trypanosomosis (tsetse-transmitted). In: Manual of diagnostic tests and vaccines for terrestrial animals (mammals, birds and bees), Vol. 1. OIE, Paris, France, 809-818

Pokou K., Kamuanga M.J.B., N'Gbo A.G.M., 2010. Farmers' willingness to contribute to tsetse and trypanosomosis control in West Africa: the case of northern Côte d'Ivoire. Biotechnol. Agron. Soc. Environ., 14 (3): 441-450
Pollock J.N., 1982. Manuel de lutte contre la mouche tsé-tsé. Vol. 1, Biologie, systématique et répartition des tsé-tsé. FAO, Rome, Italie, 310 p.

Sokouri D.P., Loukou N.E., Yapi-Gnaoré C.V., Mondeil F., Gnangbe F., 2007 Caractérisation phénotypique des bovins à viande (Bos taurus et Bos indicus au centre (Bouaké) et au nord (Korhogo) de la Côte d'Ivoire. Anim. Genet. Resour., 40: 43-53, doi: 10.1017/S1014233900002182

Solano P., Bouyer J., Itard J., Cuisance D., 2010. Cyclical vectors of trypanosomosis. In: Infectious and parasitic diseases of livestock (Lefèvre P.-C., Blancou J., Chermette R., Uilenberg G.). Lavoisier Tec \& Doc, Paris, France, 155-183

Tanenbe C., Gambo H., Musongong A.G., Boris O., Achukwi M.D., 2010. Prevalence of bovine trypanosomosis in the Faro and Deo, and Vina divisions in Cameroon: outcome of 20 years of tsetse control. Rev. Elev. Med. Vet. Pays Trop., 63 (3-4): 63-69, doi: 10.19182/remvt.10099

Torr S.J., Prior A., Wilson P.J., Schofield S., 2007. Is there safety in numbers? The effect of cattle herding on biting risk from tsetse flies. Med. Vet. Entomol., 21 (4): 301-311, doi : 10.1111/j.1365-2915.2007.00705.x

Vitouley S.H., 2005. Etude du potentiel trypanocide d'extraits aqueux de plantes médicinales pour le traitement de la trypanosomose animale africaine. Thèse Doct. Vét., Université Cheick Anta Diop, Dakar, Sénégal, 96 p.

Yapo E.M., 2011. Analyse économique de la filière du médicament vétérinaire en Côte d'Ivoire. Thèse Doct. Vét., Université Cheick Anta Diop, Dakar, Sénégal, $93 \mathrm{p}$.

Yoni W., Bila C., Bouyer J., Desquesnes M., Kaboré I., 2005. La dissection des glossines ou mouches tsé-tsé. Cirdes, Bobo Dioulasso, Burkina Faso, 12 p. (Santé animale en Afrique de l'Ouest ; 23)

Zinga Koumba R.C., Bouyer J., Mavoungou J.F., Acapovi-Yao G.L., Kohagne Tongué L., Mbang Nguema O.A., Ondo K.P.O., et al., 2013. Assessment of the diversity of bloodsucking Diptera in a swampy forest clearing of Gabon with Vavoua and Nzi traps. Rev. Elev. Med. Vet. Pays Trop., 66 (3): 91-96, doi: 10.19182/remvt.10136

\section{Summary}

Boka O.M., Boka E.E.J., Yapi G.Y., Traoré S.I., Kouamé K.E. Epidemiology of African animal trypanosomosis in cattle in Korhogo Department (Ivory Coast)

A cross-sectional study was conducted in the agropastoral zone of Kategue in Korhogo Department, Northern Ivory Coast, in order to understand better the epidemiology of African animal trypanosomosis (AAT) in cattle. The study combined an entomological survey and a parasitological survey and was conducted during the rainy season from July to October 2015. For the entomological survey, Vavoua traps were set up in 30 sites with various biotopes. For the parasitological survey, 407 cattle were sampled based on stratified random sampling without distinction of breed, sex and age. The results showed the presence of a major AAT vector, the tsetse fly Glossina palpalis gambiensis, with an overall apparent density of $0.9 \pm 3.0$ tsetse per trap per day. Only one trypanosome species was identified, Trypanosoma vivax, with relatively low prevalences in both tsetse $(11 \pm 5 \%)$ and cattle $(6 \pm 2 \%)$. Given the severity of AAT in cattle, it is necessary to raise awareness among the breeders of Korhogo, the main cattle breeding area in Ivory Coast, for the control of AAT vectors and the rational use of trypanocides.

Keywords: cattle, Glossina, trypanosomosis, epidemiology, Cote d'Ivoire

\section{Resumen}

Boka O.M., Boka E.E.J., Yapi G.Y., Traoré S.I., Kouamé K.E. Epidemiología de la tripanosomosis animal africana en ganado en el departamento de Korhogo (Costa de Marfil)

Se realizó un estudio transversal en la zona agropastoral de Kategue, en el departamento de Korhogo, al norte de Costa de Marfil, para comprender mejor la epidemiología de la tripanosomosis animal africana (TAA) en el ganado. El estudio combinó una encuesta entomológica y una encuesta parasitológica y se llevó a cabo durante la temporada de lluvias, de julio a octubre de 2015. Para el estudio entomológico, las trampas de Vavoua se instalaron en 30 sitios con varios biotopos. Para la encuesta parasitológica, se muestrearon 407 bovinos en base a un muestreo aleatorio estratificado sin distinción de raza, sexo y edad. Los resultados mostraron la presencia de un vector TAA principal, la mosca tsetse Glossina palpalis gambiensis, con una densidad aparente global de 0,9 $\pm 3,0$ tsetse por trampa por día. Sólo se identificó una especie de tripanosoma, Trypanosoma vivax, con prevalencias relativamente bajas tanto en el tsetse $(11 \pm 5 \%)$ como en el ganado $(6 \pm 2 \%)$. Dada la gravedad de la TAA en el ganado, es necesario crear conciencia entre los criadores de Korhogo, la principal área de cría de ganado en Costa de Marfil, para el control de los vectores de la TAA y el uso racional de los tripanocidas.

Palabras clave: ganado bovino, Glossina, tripanosomosis, epidemiología, Cote d'Ivoire 
